\title{
A Description of Current Curricular Design Models Used In Physical Therapy Clinical Education and Their Relationship to Pass Rates on the National Physical Therapy Examination
}

Chalee Engelhard

University of Cincinnati, engelhcr@ucmail.uc.edu

Christine McCallum

Walsh University, cmccallum@walsh.edu

Follow this and additional works at: https://nsuworks.nova.edu/ijahsp

Part of the Physical Therapy Commons, and the Physiotherapy Commons

\section{Recommended Citation}

Engelhard C, McCallum C. A Description of Current Curricular Design Models Used In Physical Therapy Clinical Education and Their Relationship to Pass Rates on the National Physical Therapy Examination. The Internet Journal of Allied Health Sciences and Practice. 2015 Oct 09;13(4), Article 5.

This Manuscript is brought to you for free and open access by the College of Health Care Sciences at NSUWorks. It has been accepted for inclusion in Internet Journal of Allied Health Sciences and Practice by an authorized editor of NSUWorks. For more information, please contact nsuworks@nova.edu. 


\title{
A Description of Current Curricular Design Models Used In Physical Therapy Clinical Education and Their Relationship to Pass Rates on the National Physical Therapy Examination
}

\begin{abstract}
Purpose. The purpose of this study was to develop descriptions and identify commonalities of current clinical education models used in physical therapy programs; then, using this information, to examine differences in first-time and ultimate pass rates among the derived formula from Commission on Accreditation in Physical Therapy Education (CAPTE) accredited programs in 2011-2013. Methods. This mixed methods study captured qualitative and quantitative data from the 204 PT programs located in the United States and Puerto Rico from 2011 to 2013. The data were coded into 11 different clinical education models which represented all programs. The models were then linked to first time and ultimate pass rates. Results. Twenty out of 204 (9.8\%) PT programs reported first-time pass rates and 201/ 204 (98.5\%) reported ultimate pass rates in the cited three-year cycle period stated in this study. The three most commonly occurring models make up $84 \%$ of all current existing models. All three frequently occurring models include both full time and clinical internship clinical experiences. Additionally, these models outperformed the national pass rate average by at least $3 \%$. Conclusions. Our results are the first to provide an analysis of the various models in use in physical therapist education, as well as a preliminary outlook on how clinical education design is matched against a program's pass rate on the national exam. Since pass rate is one of the few standardized, modifiable outcomes for CAPTE approved programs, it was selected as the outcome to allow comparison of clinical education models. One of the driving forces behind this study was to illustrate clearly the tremendous variety of clinical education models and a better understanding of the scope of the variances that is occurring.
\end{abstract}

\section{Author Bio(s)}

- Chalee Engelhard, PT, EdD, MBA, GCS, is an Assistant Professor and Director of Clinical Education, Physical Therapy, at the University of Cincinnati in Cincinnati, Ohio.

- Christine McCallum, PT, PhD, ELI Fellow APTA is a Professor and Director of Clinical Education, Physical Therapy, at Walsh University in North Canton, Ohio. 


\title{
TIAHSP \\ The Internet Journal of Allied Health Sciences and Practice
}

Dedicated to allied health professional practice and education

Vol. 13 No. 4 ISSN 1540-580X

\section{A Description of Current Curricular Design Models Used In Physical Therapy Clinical Education and Their Relationship to Pass Rates on the National Physical Therapy Examination}

\author{
Chalee Engelhard, PT, EdD, MBA, GCS 1 \\ Christine McCallum, PT, PhD, ELI Fellow APTA2
}

1. Assistant Professor and Director of Clinical Education, Physical Therapy, University of Cincinnati, Cincinnati, Ohio

2. Professor and Director of Clinical Education, Physical Therapy, Walsh University, North Canton, Ohio

United States

\begin{abstract}
Purpose. The purpose of this study was to develop descriptions and identify commonalities of current clinical education models used in physical therapy programs; then, using this information, to examine differences in first-time and ultimate pass rates among the derived formula from Commission on Accreditation in Physical Therapy Education (CAPTE) accredited programs in 2011-2013. Methods. This mixed methods study captured qualitative and quantitative data from the 204 PT programs located in the United States and Puerto Rico from 2011 to 2013. The data were coded into 11 different clinical education models which represented all programs. The models were then linked to first time and ultimate pass rates. Results. Twenty out of $204(9.8 \%)$ PT programs reported first-time pass rates and 201/204 (98.5\%) reported ultimate pass rates in the cited three-year cycle period stated in this study. The three most commonly occurring models make up $84 \%$ of all current existing models. All three frequently occurring models include both full time and clinical internship clinical experiences. Additionally, these models outperformed the national pass rate average by at least $3 \%$. Conclusions. Our results are the first to provide an analysis of the various models in use in physical therapist education, as well as a preliminary outlook on how clinical education design is matched against a program's pass rate on the national exam. Since pass rate is one of the few standardized, modifiable outcomes for CAPTE approved programs, it was selected as the outcome to allow comparison of clinical education models. One of the driving forces behind this study was to illustrate clearly the tremendous variety of clinical education models and a better understanding of the scope of the variances that is occurring.
\end{abstract}

\section{PURPOSE}

The education of physical therapists in the United States (U.S.) has two components - the classroom and the clinic. These two distinct, but connected curricula, are essential to the development of entry-level physical therapists. Doctor of Physical Therapy (DPT) students spend about $2 / 3$ of their doctoral preparation in the classroom and about $1 / 3$ in the clinic for clinical training. The number of weeks in clinic varies significantly from program to program. Since the required degree to practice as a physical therapist transitioned from masters to clinical doctorate, the number of clinical weeks required by physical therapy education programs (PTEPs) has increased 22.3\%; however, there is no evidence to support these changes. ${ }^{1}$ Upon completion of classroom and clinic educational training, DPT students sit for a national licensing exam they must pass in order to practice as a physical therapist within the U.S. Recently, leaders within the physical therapy profession have argued the state of clinical education (CE) within the United States is in a critical position. ${ }^{2}$ The changing landscape of health care, the growth of PTEPs, and the expansion of required CE weeks has strained the system. ${ }^{1,3}$

The current CE system is strained as evidenced by several factors. One source could be the imbalance of supply of clinical placements and demand by the increasing number of PT programs and students. Another possible reason for a strained CE system lies with the variability in CE curricular designs amongst the accredited programs. The Commission on Accreditation of Physical Therapy Education (CAPTE) is the nationally recognized body that accredits PTEPs. ${ }^{4}$ One of CAPTE's responsibilities 
is to establish standards by which all PTEPs are measured; however, the criteria are broad and non-prescriptive. ${ }^{4}$ Because of the wide ranging possibilities given by CAPTE during curricular design, PTEPs have latitude to develop a curriculum that includes didactic and clinical components that best meet the vision, mission, and goals of the program. That being said, CAPTE does require a minimum of 30 weeks of full time CE but does not have a maximum number of weeks. ${ }^{4}$

A common phrase heard in the physical therapy educational community is "if you've seen one physical therapy program, then you've seen only one physical therapy program," suggesting the similarities in design are few. Currently, there are different ideas about how to design a curriculum for PTEPs, and in particular, the CE curricula. Curricula can be designed based upon theories, social conditions of the day, and/or values and experiences of the professional faculty doing their best to train the next generation of physical therapists. ${ }^{5}$ The curricular designer(s) for each program determine the curriculum plan for the learners, which "includes objectives, content, learning experiences, and evaluation methods-all of which are grounded in the mission,

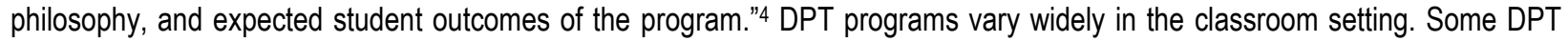
programs are hybrid with a split between online and classroom learning. There are also differences between traditional lecture based learning and problem based learning. However, no standardization of classroom curricular designs is required or present in PTEPs.

In specific regard to clinical education curricula, designers select from integrated clinical experiences, full or part time clinical education experiences, or clinical internships for which to deliver the active, applied learning experiences for learners. ${ }^{6}$ Refer to Table 1 for the definitions for clinical experiences as they apply to this study. However, no standardization of CE curricular designs is required or present in PTEPs.

Recently, Hakim et al wrote on the benefits of the integrated model of clinical education. ${ }^{7}$ Integrated clinical education curricular designs can assume a variety of structural models, including part-time or full-time experiences, to meet the intended outcome of the experience. The CE experiences are offered throughout the curriculum in a systematic and structured manner. The authors argued integrated experiences are beneficial for student learning to assist assimilation of classroom learning to authentic real life situations and build a solid foundation for development of clinical reasoning skills. Integrated clinical experiences create a wellcoordinated clinical curriculum and strengthen partnerships with local and regionally based clinical instructors for the benefit of the learner and the program.

On the other hand, Rapport et al presented the benefits for a yearlong internship CE curricular model. ${ }^{8}$ This model offers a final full time clinical education experience over the course of the final year of a learner's education. The authors reasoned the increased time in a clinical setting is needed for learners to meet expected levels of competence in today's changing health care systems. The yearlong internship creates a culminating clinical experience for the learner to apply all didactic content under the direct supervision of a clinical instructor, yet still connected to the PTEP for guidance and direction as needed.

While both models provide support for its innovative curricular design based upon current experiences in some PTEPs as well as other health professions, it is still unknown if one model is more prevalent in use than another in present day physical therapist entry-level education. ${ }^{7-8}$ It is also unclear how the placement of clinical education within the program curriculum, or the curricular design of clinical education, may impact successful outcomes on the National Physical Therapy Exam (NPTE). ${ }^{9-12}$ Jette et al eludes that with the move to the doctorate degree in physical therapy, many programs created a curricular structure which placed full time CE experiences towards the end of the curriculum; however, no aggregated evidence was found to support this curricular move. ${ }^{2}$

Thus, there is a critical gap between current practice and the evidence to support new variations of clinical education models in response to the ever-evolving health care landscape. PTEPs need to respond to the perceived imbalance between supply of clinical sites and instructors with the demand of PTEPs for placements of their students. Knowledge of existing educational practices within the profession of physical therapy may assist in determining best practice for future outcomes. As a result, the purpose of this study was to develop descriptions and identify commonalities of current clinical education models used in PTEPs. Then, taking this information and examine differences in performance on the NPTE as it relates to first-time and ultimate pass rates among the derived formulas, among all CAPTE accredited programs from 2011-2013. In accordance with CAPTE and this study, first-time pass rates are defined as the percent of DPT students who pass the NPTE on their first attempt and the ultimate pass rate refers to the percent of students in a graduation class that took the NPTE and passed, no matter how many attempts it took. ${ }^{4}$

(C) The Internet Journal of Allied Health Sciences and Practice, 2015 


\section{METHODS}

\section{Design and Setting}

This study's mixed methodology was selected in order to provide a framework for the coding of data; uncovering potential trends; and determining level of support for a quantified data set. Grounded theory was used as the qualitative methodology recognizing that Strauss and Corbin's later work with grounded theory supported analyzing trended data without ultimately seeking achievement of a central category. ${ }^{14}$ Thus, this study's qualitative approach is in line with grounded theory where a nonquantitative approach is used to interpret data and discover concepts and relationships between data sets. ${ }^{14}$ Therefore, in this study, without the open and axial coding there would not have been an ability to trend the data to uncover commonly used models.

Thus, this study's research design captured qualitative and quantitative data from the 204 PT programs (includes both DPT and MPT) located in the United States and Puerto Rico from 2011 to 2013. Qualitative and quantitative primary datasets were gleaned using the same methods; the APTA and PTEP websites, and/or follow-up individual personal contact with a program representative. The data gleaned from each program included curricular descriptions, clinical education rotation classifications such as part time or full time, integrated or terminal, total number of weeks of clinical education, length and total number of rotations, and NPTE first time and ultimate pass rates.

Because of the method for gathering the qualitative data, a link to individual programs was possible. The quantitative primary data set used a three-year period to examine pass rates, 2011-2013.The secondary dataset was derived from an open access FSBPT spreadsheet that provided ultimate pass rate information on each PTEP in the US and Puerto Rico. This spreadsheet was accessed through the CAPTE website. ${ }^{15,17,18}$ This secondary dataset was introduced into the quantitative methods to link ultimate pass rates to individual programs. See Figure 1 for an illustration of how the qualitative and quantitative data originated.

\section{Figure 1. Illustrate Origin of Quantitative and Qualitative Data}

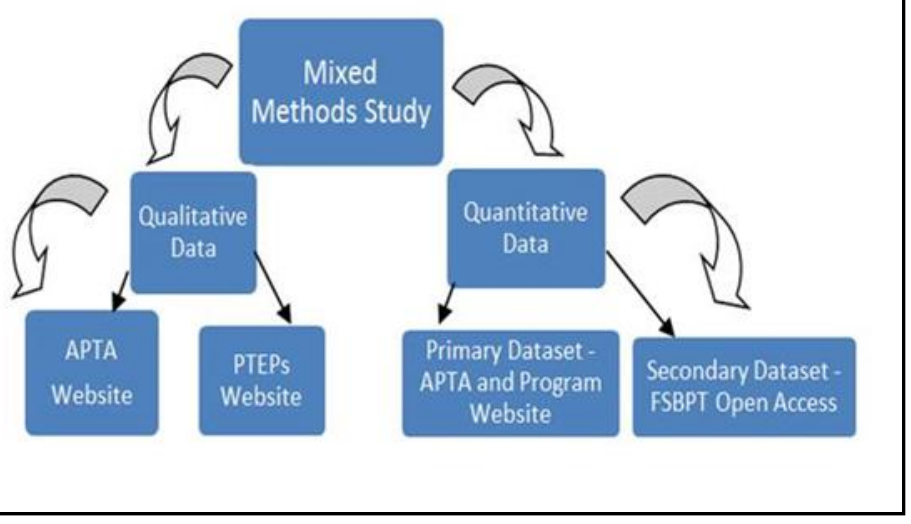

The study was submitted to the Institutional Review Board of the University of Cincinnati, Cincinnati, Ohio, (study ID, 2013-3151) where it was determined to not require full review and approval, as it was not human subject research.

\section{Qualitative: Methods, Dependability, Trustworthiness and Data Analysis.}

Methods. Qualitative data yielded unique, clinical education models through examination of the types of clinical experiences provided by each PT program. Most clinical experience information was collected through examination of the program websites. When the website information was unclear or insufficient for this study's purposes, DPT programs were contacted via email or telecommunications to attempt to complete the data set. The categories used for the clinical education experiences were integrated, part time, full time, and clinical internship. Most of the definitions used to create each of the categories were those shared with the PT community via the American Council of Academic Physical Therapy (ACAPT), as seen in Table 1.16 
Table 1. ACAPT Definitions for Clinical Education Rotation Categories

\begin{tabular}{|l|l|}
\hline Type of Rotation & Definition \\
\hline Integrated Clinical Experience & $\begin{array}{l}\text { A clinical learning experience that occurs in an integrated fashion concurrently with did actic course work } \\
\text { in which student physical therapists are learning in clinical environments }\end{array}$ \\
\hline $\begin{array}{l}\text { A clinical learning experience that is from } 5 \text { weeks long or less weeks in length in which student physical } \\
\text { therapists are learning in clinical environments. Stud ents will return to further didactic learning } \\
\text { experiences following completion of this clinical experience. }\end{array}$ \\
$\begin{array}{l}\text { A clinical learning experience that is from six to twelve weeks in length in which student physical } \\
\text { therapists are learning in clinical environments. Stud ents will return to further didactic learning } \\
\text { experiences following completion of this clinical experience. }\end{array}$ \\
\hline $\begin{array}{l}\text { The extended full-time clinical education experience(s) typically following the completion of the didactic } \\
\text { coursework for the Doctor of Physical Therapy degree. The clinical internship (s), in total, is from three } \\
\text { months to twelve months in length. The clinical internship may extend beyond graduation and continue } \\
\text { as a post-licensure experience as part of the preparation of Doctors of Physical Therapy. }\end{array}$
\end{tabular}

For this study, the only definition that was altered from ACAPT standardized terminology was clinical internship. The ACAPT definition states that clinical internships occur after all didactic work and are 3 to 12 months long. ${ }^{6}$ The definition was altered in order to accommodate for rotations that were less than 3 months long but still occurred after all didactic work was completed. This accounted for 101 PTEPs. Thus, for this study, a clinical internship was defined as a clinical rotation that occurred after all didactic work was completed, was full time, and was at a minimum 7 weeks in duration.

Dependability and trustworthiness. To enhance the dependability (reliability) and trustworthiness of the qualitative results, the research team took purposeful steps. Analysis of the coding procedure allowed for dependability through transparency of coding approaches. The researchers independently coded $10 \%$ of the dataset, then met to confirm coding accuracy, whereby $100 \%$ agreement was achieved in coding schema. Additionally, the researchers intentionally reveal an extensive description of the research process with a focus on procedures and limitations so that a consumer of the research can have increased confidence in the study..$^{19}$

Data analysis. Using grounded theory, the initial open qualitative coding attempt resulted in 19 different clinical education models out of examination of the first 25 PT programs simply by assessing the number of offered rotations and clinical education categories e.g., 2 part time experiences +2 full time clinical experiences +3 clinical internships. ${ }^{14}$ This recognition resulted in the development of a consistent coding approach through grounded theory method that used categorical types of rotations rather than the quantitative number of rotations offered by any individual program. Thus, through further open coding, the above example transitioned to -- part time experiences + full time experiences + clinical internships. From here, the model formulas were axial coded into nominal data. Thus, the researchers chose not to look at coding by specific number of occurrences of each clinical experience because of the number of variations in program formulas. This decision promoted the ability to provide a consistent coding paradigm.

\section{Quantitative: Measurements and Data Analysis}

Measurements. Out of 204 PTEPs, the researchers found on PTEP and APTA websites only 74 (36\%) PTEPs reported first-time pass rates whereas $146(72 \%)$ programs reported ultimate pass rates. Unfortunately, the year(s) used to report pass rate data varied significantly; therefore, in order to examine consistent pass rate outcomes, only PTEPs who supplied 3-year pass rate outcomes since 2011 were used. ${ }^{17}$ This reduced the dataset to 20 PTEPs for first time pass rate and 45 for three year ultimate pass rate. A secondary data set was used to determine ultimate pass rate for the representative programs in the United States and Puerto Rico for the years of 2011-2013.15 No open access secondary data set exists for first time pass rate, as the Federation of State Boards of Physical Therapy (FSBPT) state that ultimate pass rate is a better measurement of a PTEP's status.$^{87}$ Continued inclusion of the first time pass rate data in this study occurred since first-time and ultimate pass rates are frequently reported together in the literature and these outcomes contribute to the criteria that many PTEPs use to evaluate the success of their program.

Data analysis. Thus, frequency of the most commonly occurring clinical education models allowed for ranking of the models by commonality. Analysis of program-reported first-time and ultimate pass rates occurred using the same criteria as the qualitative; thus, first-time and ultimate pass rates data were used only if the PTEP reported the pass rate outcome within the period of 2011 to 2013. Comparison of PTEPs that were found to have the most commonly used clinical education models occurred by calculating the mean of the first-time and ultimate pass rates. 


\section{RESULTS}

Qualitative: Clinical Education Curriculum Design Models

The clinical education formulas enabled coding of 11 different models that were in use by CAPTE accredited PT programs in 2013 as seen in Table 2.

Table 2. Commonly Occurring Clinical Education Models

\begin{tabular}{|l|c|c|}
\hline Codes/Model Type & Frequency & Percentages \\
\hline $1=$ Integrated + Part time + Full time + Clinical Internship & 7 & $3 \%$ \\
\hline $2=$ Integrated + Full time + Clinical Internship & 27 & $13 \%$ \\
\hline $3=$ Integrated + Clinical Internship & 6 & $3 \%$ \\
\hline $4=$ Part time + Full time + Clinical Internship & 70 & $34 \%$ \\
\hline $5=$ Part time + Clinical Internship & 9 & $4 \%$ \\
\hline $6=$ Full time + Clinical Internships & 75 & $37 \%$ \\
\hline $7=$ Clinical Internships only & 3 & $1 \%$ \\
\hline $8=$ Integrated + Part time + Clinical Internships & 4 & $2 \%$ \\
\hline $9=$ Full time & 1 & $0 \%$ \\
\hline $10=$ Part time + Full time & 1 & $0 \%$ \\
\hline $11=$ Integrated + Full time & 1 & $0 \%$ \\
\hline Total: & 204 & \\
\hline
\end{tabular}

The top three most commonly occurring clinical education design models were formula codes "6", "4", and "2" in descending order.

Table 3. Most Commonly Occurring Models

\begin{tabular}{|l|c|r|}
\hline & Frequency & Percentages \\
\hline $6=$ Full time + Clinical Internships & 75 & $37 \%$ \\
\hline $4=$ Part time + Full time + Clinical Internship & 70 & $34 \%$ \\
\hline $2=$ Integrated + Full time + Clinical Internship & 27 & $13 \%$ \\
\hline
\end{tabular}

These three models comprise $84 \%$ of all currently existing models. Note that all three frequently occurring models include both full time and clinical internship clinical experiences. On the other hand, the least frequent clinical education models were codes "9", "10", and "11" in descending order as seen in Table 4.

Table 4. Least Commonly Occurring Models

\begin{tabular}{|l|c|c|}
\hline & Frequency & Percentages \\
\hline $9=$ Full time & & 1 \\
\hline $10=$ Part time + Full time & 1 & $0 \%$ \\
\hline 11 =Integrated + Full time & 1 & $0 \%$ \\
\hline
\end{tabular}

All three models included full time experiences and none had clinical internships. These least commonly occurring models in summation made up only $3 \%$ of the models in current use.

\section{Quantitative: Link of pass rate to NPTE program outcomes \\ First time pass rate}

Twenty out of $204(9.8 \%)$ PTEPs reported first-time pass rates in a three-year cycle with a cited three-year period of 2011 to 2013. With first time pass rates and ultimate pass rates frequently being cited together in research and first times pass rates contributing to the criteria that many PTEPs use to evaluate the success of their program, its inclusion in this study remained. Thus, due to a low $N$, we examined only those programs with two of the common CE design models, (coded "4" or "6"), to determine a mean first time pass rate. The linked first-time pass rates to these specific coded clinical education models:

- $\quad$ "4" code first-time pass rate from 4 programs: $95.93 \%$

- "6" code first-time pass rate from 11 programs: $94.68 \%$ 


\section{Ultimate pass rate}

Thirty out of $204(14.7 \%)$ programs reported ultimate pass rates in the three-year cycle on the APTA or program website within a three-year period of 2011 to 2013 . The linked ultimate pass rates to these specific coded clinical education models were:

- $\quad 4 "$ code ultimate pass rate from 10 programs: $98.75 \%$

- $\quad$ "6" code ultimate pass rate from 20 programs: $95.87 \%$

From the secondary data set, 201/204 (98.5\%) PTEPs in the United States and Puerto Rico reported ultimate pass rates, which enabled a deeper analysis of all three commonly occurring models. By matching the coded programs from the primary data that came from APTA and program websites to the secondary dataset that came from the CAPTE website, the researchers were able to calculate more credible ultimate pass rates, as follows:

- $\quad$ "2" code ultimate pass rate: $97.17 \%$

- $\quad$ "4" code ultimate pass rate: $98.27 \%$

- " 6 " code ultimate pass rate: $96.44 \%$

Additionally, when considering the " 6 " code PTEPs, there was one program that was an outlier by 23 percentage points where its ultimate pass rate was $50 \%$. When this program's ultimate pass rate was removed from the calculations, the modified ultimate pass rate for "6" coded PTEPs became $97.08 \%$.

\section{CONCLUSIONS AND RECOMMENDATIONS Wide variety of models}

First, our results reveal a wide variety of clinical education curricular design models exist throughout entry-level physical therapist education programs (2011-3013). The simple exercise of coding the clinical education models demonstrates a variety in the overall curricular design of CE in PT education. While we coded 11 different models, $84 \%$ of programs were described by one of three models: 1) integrated, full time and clinical internships, 2) part time, full time, and clinical internship, or 3) full time and clinical internship. What these results tell us in that $84 \%$ of programs offer at least a 7 week clinical education experience at the end of the curriculum (per our definition of a clinical internship), and also either integrated part time or full time experiences. The results also reveal that less than $1 \%$ of programs use a yearlong, terminal experience. Lastly, the range of weeks for final rotations in these datasets was 7 to 52 weeks. Martorello found that the preference for a final clinical was on average $9.1(+/-$ 2.09) weeks with a range of 5.5-16 weeks; however, no definitive conclusions were reached. ${ }^{20}$ Our results confirm that a firm concensus for the number of weeks for a final clinical education experience currently does not exist in entry-level clinical eduction.

It is understandable, when looking at these variations, why a call for standardization may be necessary from a structural viewpoint.2, 7-8, 21-22 If curriculums are designed based upon theory, social conditions of the day, and/or values of the faculty, then there is now evidence for support of clinical education curriculum design models for clinical internships coupled with either part time, full time, or integrated experiences. However, there is less evidence to support yearlong internship models as well as models that incorporate a longer clinical experience (longer than 16 weeks) at the end of PTEP curriculums. This is verified by the infrequency of use of clinical experiences that span longer than a usual academic semester, as such, the ability to standardize clinical education curricular design for large scale use.

\section{Impact of pass rates}

Second, we were interested in determining if pass rate on the NPTE would vary depending on the clinical education curricular design models. This was a difficult task, as the number of programs that reported first-time (9.8\%) and ultimate pass rate (14.7\%) on the APTA and program websites was low. FSBPT states that first-time pass rates are not indicative of a PTEP's overall performance and is not a standard outcome measure required of reporting to the public. ${ }^{18}$ As such, we were not surprised that the majority of PTEPs did not share this data with the public. We were concerned however, with the inconsistent reporting on PTEP program websites about ultimate three year pass rates as this is a requirement. ${ }^{18}$ Therefore, using the ultimate pass rate secondary dataset available from CAPTE brought validity to the study.

For the three most commonly occurring clinical education curricular design models (codes $2,4,6$ ), the first time pass rate was $95.98 \%$ and ultimate pass rate was $97.29 \%$. These rates are higher than the national average for 2013 first-time and ultimate pass rate $\left(87.7 \%\right.$ / $94.20 \%$ respectively). ${ }^{17,23}$ The ultimate pass rate for the least commonly occurring models (codes $\left.9,10,11\right)$, was almost $3 \%$ less than the national average. Although a comparison for first time pass rates with the low $\mathrm{N}$ is not warranted, a comparison for ultimate pass rates is. The most common CE models in the US have higher pass rates than the less commonly used models. Upon examining the CE models with the lowest reported pass rates, national pass rate averages were lower than programs with the commonly occurring CE curricular models that included both full time experiences and clinical internships.

(c) The Internet Journal of Allied Health Sciences and Practice, 2015 


\section{What could this mean?}

Finally, because of the diversity of curricular designs, it could be suggested that changing the current clinical education practice may be difficult. Clinical education curricular redesign would require extensive programmatic changes, even for programs that already exhibit successful outcomes on the NPTE. Since pass rate is one of the few standardized, modifiable outcomes for CAPTE approved programs, it was selected as the outcome to allow comparison of clinical education models.

PTEPs strive to meet and surpass the current CAPTE criteria in order to not only maintain accreditation but also to graduate well-prepared physical therapists. Additionally, the stakeholders that support clinical education have displayed a history of tolerance for these inconsistencies. ${ }^{24}$ However, there have been a multitude of changes in recent times with the turn to entry level doctoral training, including increasing productivity standards and the healthcare industry's response to the Affordable Care Act, that have created this call for standardization. ${ }^{25}$ One of the driving forces behind this study was to illustrate clearly the tremendous variety of clinical education models and to help provide a foundation to facilitate obtaining answers for the call of standardization from our profession. This will also offer a better understanding of the scope of the task at hand.

\section{Limitations}

It is important to acknowledge the limitations of this study. The use of websites to glean data and use of public documents are at the center of the limitations for this study. The content available for review on a PTEP website was used as the basis for data collection about the CE curriculum. While we standardized the data collection dates and data gleaned about the program, the information on university webpages had the potential to be outdated as we were not able to locate the publication date for each program, thus the potential for measurement bias exists. ${ }^{26}$ While the potential for measurement bias exists, we believe the results provide a general description of the type of clinical education curricular models that were in use in entry-level physical therapy education from the years 2011-2013.

Another limitation involves the inclusion of the first time pass rate data in the study despite its small sized dataset. We decided to include first time pass rate as an outcome variable; however, a low number of PTEPs actually include this program outcome for public review. Because of this small dataset, sampling bias exists. ${ }^{26}$ These results should not be generalized to the larger population to compare clinical education curricular design models and program success as measured by first time pass rate on the NPTE. Our study also did not examine statistical significance between the clinical education curricular design models and three year pass rate; therefore no generalizability should be made from this study. Our purpose was to solely examine trends in how design could impact an outcome.

\section{Future research}

With respect to future research, we believe further investigation is needed to examine pass rates and the most common CE curricular design models with clarification of class room design for a better overall understanding of the program. A study using a stratified sample may help demonstrate a link between curricular design and program outcomes, such as pass rate. Where this study provides a much needed foundation for acknowledging the types of models in current use and a rudimentary link between models and outcomes, there is a need to determine the efficacy of the different models. The physical therapy profession must ensure that CE models provide the best learning experience for the varied learning styles students have. It is possible that clinical partners may not tolerate the wide variety of CE models in the physical therapy profession.

As Jette et al stated, the physical therapy profession must use what is known and then move along as best we can, keeping in mind the social conditions of the day and the changes to the health care environment is what is pushing for the change, rather than a true need to change a curriculum because students are not prepared. ${ }^{2}$ We need to change because the external environment has changed so much to meet the needs of the learner. Other countries have faced similar situations and have gone forward with making significant changes to their standard modes of operation without necessarily having evidence to support the chosen paths. ${ }^{27-30}$

The purpose of this research study was to describe the curricular design models used in PT clinical education, to ascertain the frequency of use, and to determine a rudimentary relationship between CE curricular design and program outcomes relating to pass rate on the NPTE. Jette et al reported that the physical therapy profession needs to decide if change to current practice is warranted given the lack of purposeful evidence to support that change and questioned if CE structure contributes to student/program outcomes. ${ }^{2}$ This study responded to these points. Our results are the first to provide an analysis of the various models in use in physical therapist education, as well as a preliminary outlook on how clinical education design is matched against a program's pass rate on the national exam.

(c) The Internet Journal of Allied Health Sciences and Practice, 2015 


\section{REFERENCES}

1. McCallum CA, Mosher PD, Howman J, Engelhard C, Euype S, Cook CE. Development of regional core networks for the administration of physical therapist clinical education. JOPTE. 2014;28(1):39-47.Jette

2. DU, Nelson L, Palaima M, Wetherbee E. How do we improve quality in clinical education? Examination of structure, processes and outcomes. JOPTE. 2014;28(1):6-10.

3. Applebaum D, Portney LG, Kolosky L, McSorley O, Olimpio D, Pelletier D, Zupkus M. Building physical therapist education networks. JOPTE. 2014;28(1):30-8.

4. Commission on Accreditation Physical Therapy Education. Evaluative Criteria for Accreditation of Education Programs for the Preparation of Physical Therapists. American Physical Therapy Association Web site.

http://www.capteonline.org/uploadedFiles/CAPTEorg/About_CAPTE/Resources/Accreditation_Handbook/EvaluativeCri teria_PT.pdf. 2011. Accessed October 1, 2014.

5. Jensen G, Mostrom E. Handbook of Teaching and Learning for Physical Therapists, 3e. Elsevier Health Sciences. 2013.

6. Academic Council of the American Physical Therapy Association. Position: New physical therapist academic programs. http://acaptaonline.org/pdf/2012motins/AC\%20201\%20Motion\%20Developing\%20PT\%20Programs.pdf. Accessed October 1, 2014.

7. Wruble-Hakim E, Moffat M, Becker E, Bell KA, Manal TJ, Schmitt L, Ciolek C. Application of educational theory and evidence in support of an integrated model of clinical education. JOPTE. 2014;28(1):13-22.

8. Rapport MJ, Kelly MK, Hankin TR, Rodriguez JW, Tomlinson SS. A shared vision for clinical education: the year long internship. JOPTE. 2014;28(1):22-9.

9. Mohr T, Ingram D, Hayes S, Du Z. Educational program characteristics and pass rates on the national physical therapy examination. JOPTE. 2005;19:60-6.

10. Zahn CM, Saquil A, Artino AR, et al. Correlation of national board of medical examiners scores with United Stated medical licensing examination step 1 and step 2 scores. Acad Med. 2012;87:1348-54. [PMID:22914528]

11. Riddle D, Utzman RR, Jewell DV. Academic difficulty and program-level variables predict performance on the national physical therapy examination for licensure: a population-based cohort study. Phys Ther. 2009;89:1182-91.

12. Thieman TJ, Weddle ML, Moore MA. Predicting academic, clinical, and licensure examination performance in a professional (entry-level) master's degree program in physical therapy. JOPTE. 2003;17:32-7.

13. Utzman RR, Riddle DL, Jewell DV. Use of demographic and quantitative admissions data to predict performance on the national physical therapy examination. Phys Ther. 2007;87:1181-93.

14. Strauss A, Corbin J. Basics of qualitative research. London, England: Sage Publications, 1998.

15. CAPTE Website, http://www.capteonline.org/AAR/ Accessed July 16, 2014.

16. American Council of Academic Physical Therapy. AC-2-13: Terminology for Clinical Education Experiences. Available at: http://www.acapt.org/images/AC-2-13 Terminology for_Clincal_Education_PASSED.pdf Accessed August 5, 2015.

17. APTA website, http://www.apta.org/apta/directories/accreditedschools.aspx?navlD=10737423273 Accessed July 16, 2014.

18. FSBPT - Ultimate pass rate publication. https://www.fsbpt.org/FreeResources/NPTEPassRateReports.aspx Accessed October 2, 2014.

19. Maykut $P$, Morehouse R. Beginning qualitative research: $A$ philosophic and practical guide. London: Falmer Press, 1994.

20. Martorello L. The optimal length of clinical internship experiences for entry-level physical therapist students as perceived by center coordinators of clinical education: a pilot study. J Phys Ther Educ. 2006;20(1):56-8.

21. Higgs J. Managing clinical education: the programme. Physiotherapy. 1993;79(4):239-46.

22. Wetherbee E, Peatman N, Kenney D, Cusson M, Applebaum D. Standards for Clinical Education: A Qualitative Study. JOPTE. 2010;24(3):35.

23. http://www.apta.org/licensure/npte/ First-time pass rate for cycle of 2010-2012. Accessed July 16, 2014.

24. Gwyer J, Odom C, Gandy J. History of clinical education in physical therapy in the United States. J Phys Ther Educ. 2003;17(3):34-43.

25. Recker-Hughes C, Wetherbee, E, Buccieri K, Timmerberg J, Stolfi A. Essential Characteristics of Quality Clinical Education Experiences: Standards to Facilitate Student Learning. JOPTE. 2014;28(1):48-55.

26. University of Chicago at Illinois. Research bias. Available at: http://tigger.uic.edu/ /wbenn/jacswcourses/socw360/week14. Accessed October 1, 2014.

27. Smith PM, Corso LN, Cobb N. The perennial struggle to find clinical placement opportunities: a Canadian national survey. Nurse Educ Today. 2010;30(8):798-803. [PMID: 20378214] 
28. National Association for Clinical Education in Physiotherapy. Clinical education and placements. Canadian Council of Physiotherapy University Programs website. http://www.physiotherapyeducation.ca/ClinicalEducation.html. Accessed September 9, 2013.

29. Dean CM, Stark AM, Gates CA, et al. A profile of physiotherapy clinical education. Aust Health Rev. 2009;33(1):39-46. [PMID: 19203332]

30. Norman KE, Booth R, Chisholm B, et al. Physiotherapists and physiotherapy student placements across regions in Ontario: a descriptive comparison. Physiother Can. 2013;65(1):64-73. [PMID: 24381384] 